\title{
Emergency physicians are more accurate in detecting pulmonary embolism at the emergency department then internal medicine physicians
}

\author{
Ulf Martin Schilling \\ From Danish Society for Emergency Medicine: Research Symposium 2010 \\ Roskilde, Denmark. 20-21 May 2010
}

\section{Background}

Detecting pulmonary embolism (PE) is a major problem at the ED. Final diagnosis often is made by CT-scan. Due to specialisation in emergency medicine emergency physicians might perform better than internal medicine physicians in detecting PE. To confirm this hypothesis, a single center retrospective cohort-study was performed.

\section{Methods}

During the three-month periods (march till may) 2007 and 2008 the findings in all patients undergoing pulmonary $\mathrm{CT}$ at our emergency department were reviewed. The investigations were attributed to emergency physician (EP) or internal medicine physician (IP). Negative and positive investigations were evaluated, and the number of patients treated by the respective group were calculated. Statistical analysis was performed by the Students-T-test, and probability levels of $5 \%$ were accepted as significant.

\section{Results}

In 2007, a total of 2847 patients attended for medical problems. 576 were treated by EP (20.23\%). In 2008, 2408 patients searched for medical problems and 625 (25.95\%) were attended by EP. EP ordered a total of 34 pulmonary CT 2007 and 35 in 2008. 17.64\% (2007)/ $22.86 \%$ (2008) of these resulted in the diagnosis of PE. IP ordered a 77 (2007) and 64 (2008) pulmonary CT, with positive findings in $12.98 \%$ (2007)/10.93\% (2008). EP ordered pulmonary CT for $5.9 \%$ (2007)/5.6\% (2008) of their patients, whilst IP performed CT-scans in 3.39\% $(2007) / 3.59 \%(2008)(\mathrm{p}=0.0108)$. This means that EP have a higher index of suspicion for the diagnosis of $\mathrm{PE}$ at the emergency department (1.74 (2007)/1.56 (2008).

CT-scans were positive in $1.04 \%$ (2007)/1.28\% (2008) for the EP, and in $0.43 \%$ (2007)/0.39\% (2008) for the IP $(\mathrm{p}<0.01)$. EP are more accurate in detecting PE at the emergency department (OR 2.43(2007)/3.26(2008).

At the hospital including the emergency department were 14.29\% (2007)/14.15\% (2008) of CT-scans positive. No significant difference could be found between the positive findings for all the hospital compared to the EP $(\mathrm{p}=0.38)$ or IP $(\mathrm{p}=0.27)$.

\section{Conclusion}

Emergency physicians seem to have a higher index of suspicion for PE than internal medicine physicians and are more accurate in detecting PE at the emergency department. Compared with the total of our university hospital, emergency physicians are at least comparable in diagnosing PE.

Published: 17 September 2010

doi:10.1186/1757-7241-18-S1-P13

Cite this article as: Schilling: Emergency physicians are more accurate in detecting pulmonary embolism at the emergency department then internal medicine physicians. Scandinavian Journal of Trauma,

Resuscitation and Emergency Medicine 2010 18(Suppl 1):P13. 